\title{
Disorder Induced BCS-BEC Crossover in an Ultracold Fermi Gas
}

\author{
Ayan Khan · Saurabh Basu • B. Tanatar
}

Received: 8 November 2012 / Accepted: 1 December 2012 / Published online: 22 December 2012

(C) Springer Science+Business Media New York 2012

\begin{abstract}
We develop the formalism for BCS-BEC crossover in the presence of weak random impurity and calculate the effect of the random potentials on the basic mean-field quantities. The disorder has been included through the Nozières and Schmitt-Rink theory of superconducting fluctuations, and we obtain the disorder induced superfluid order parameter and chemical potential through a self-consistent calculation. We also calculate the condensate fraction which reveals a distinct nonmonotonic behavior. The downturn in the latter result occurs at the crossover regime with gradual depletion on the BEC side. The non-monotonic feature in the condensate fraction data has been measured in clean systems. Motivated by the above result, we discuss the stability of a disordered fermionic superfluid in the crossover regime.
\end{abstract}

Keywords Superconductivity - BCS-BEC crossover . disorder effects

\section{Introduction}

The smooth evolution of atoms from Bardeen-CooperSchrieffer (BCS) physics governed by Cooper pairs to

\footnotetext{
A. Khan $(\bowtie)$

Indian Institute of Science Education and Research (IISER)

Kolkata, Mohanpur Campus, Nadia 714252, India

e-mail: ayankhan@gmail.com

S. Basu

Department of Physics, IIT Guwahati, Guwahati, Assam 781039, India

B. Tanatar

Department of Physics, Bilkent University, Bilkent,

06800 Ankara, Turkey
}

composite bosons which have undergone Bose-Einsteincondensation (BEC) is a very exciting domain of research in recent years. Such a transformation of ultracold atomic gases can easily be realized by changing inter-atomic low energy scattering length by means of Fano-Feshbach resonance [1]. The experimental advances in cooling and trapping techniques have introduced the possibility for studying the BCS-BEC crossover more closely [2, 3].

Recent developments in the experimental front allows one to look at a more intriguing aspect in ultracold atomic gases, namely the effect of disorder. In the seminal work of Anderson [4], it was shown that beyond some critical amount of impurity in the electronic system, electrons get localized in space. But a direct observation of Anderson localization in electronic systems is very difficult. On the other hand, ultra-cold atoms make it possible to address the core of the phenomenon that Anderson had discovered (exponential decay of the wavefunction), since they are genuine quantum particles described as matter waves. The biggest advantage of using ultracold atomic systems is the high level of controllability and tunability of interaction (through external magnetic field) and disorder (by means optical arrangements). Recent experimental studies in Bose and Fermi systems [5-8] have provided further impetus on the issue of disorder in cold atomic gases [9]. These investigations have widened the possibility to study the crossover in the light of disorder experimentally.

Theoretically, the static disorder in Fermi and in Bose systems has been studied in the context of dirty superconductors [10-12] and Bose condensates [13, 14]. The last decade has seen an enormous growth in the study of disorder in Bose gas (see, for instance, $[5,6,9,18])$. The interest in systems at unitarity is also gaining pace [15-18].

In this work, we investigate the BCS-BEC crossover with weak uncorrelated disorder at zero temperature. We have 
followed the prescription of $[15,16]$ which is actually connected with Nozières and Schmitt-Rink (NSR) theory [19] of superconducting fluctuations. This enables us to solve the modified density and gap equations with disorder as input parameter self-consistently. We extend our analysis further to compute condensate fraction which reveals an interesting non-monotonic behavior and we discuss this result in the light of the experimental observation of condensate fraction in a clean Fermi gas [20].

We organize the rest of this paper as follows. In Section 2, we summarize the basic formalism of $[15,21]$ to include fluctuations at the mean-field level. Our results are presented in Sect. 3, and we close with a conclusion in Sect. 4.

\section{Model and Theory}

To describe the effect of impurity in a Fermi superfluid in the crossover from BCS to BEC regime, one needs to start from the real space Hamiltonian in three-dimensions for a $s$-wave superfluid,

$$
\begin{aligned}
\mathscr{H}(\mathbf{x})= & \sum_{\sigma} \Phi_{\sigma}^{\dagger}(\mathbf{x})\left[-\frac{\nabla^{2}}{2 m}-\mu+V_{d}(\mathbf{x})\right] \Phi_{\sigma}(\mathbf{x}) \\
& +\int d x^{\prime} V\left(\mathbf{x}, \mathbf{x}^{\prime}\right) \Phi_{\uparrow}^{\dagger}\left(\mathbf{x}^{\prime}\right) \Phi_{\downarrow}^{\dagger}(\mathbf{x}) \Phi_{\downarrow}(\mathbf{x}) \Phi_{\uparrow}\left(\mathbf{x}^{\prime}\right),
\end{aligned}
$$

where $\Phi_{\sigma}^{\dagger}(\mathbf{x})$ and $\Phi_{\sigma}(\mathbf{x})$ represent the creation and annihilation of fermions with mass $m$ and spin state $\sigma$ at $\mathbf{x}$, respectively. $\mathscr{V}_{d}(\mathbf{x})$ denotes the random impurity potential, and $\mu$ is the chemical potential. We set the Planck's constant $\hbar=1$. The $s$-wave fermionic interaction is defined by $\mathscr{V}\left(\mathbf{x}, \mathbf{x}^{\prime}\right)=$ $-g \delta\left(\mathbf{x}-\mathbf{x}^{\prime}\right)$, and $g$ is the bare coupling strength of fermionfermion pairing. We choose the disorder as uncorrelated; therefore, the range of the impurity should be much smaller than the average separation between them. The disorder potential is modeled as $\mathscr{V}_{d}(\mathbf{x})=\sum_{i} g_{d} \delta\left(\mathbf{x}-\mathbf{x}_{i}\right)$ where $g_{d}$ is a fermionic impurity coupling constant and $\mathbf{x}_{i}$ are the static positions of the quenched disorder. The correlation function turns out to be $\left\langle\mathscr{V}_{d}(-q) \mathscr{V}_{d}(q)\right\rangle=\beta \delta_{i v_{m}, 0} \kappa$ while $q=\left(\mathbf{q}, i v_{m}\right) . \beta$ is the inverse temperature, $v_{m}$ is the bosonic Matsubara frequency $\left(v_{m}=2 \pi m / \beta\right.$, with $m$ being an integer) and $\kappa=n_{i} g_{d}^{2}$, and $n_{i}$ is the impurity concentration.

The partition function corresponding to the Hamiltonian in Eq. (1) can be written in the path integral formulation as

$$
\mathscr{Z}=\int \mathscr{D}[\bar{\Phi}, \Phi] \exp [-\mathscr{S}(\{\bar{\Phi}\}\{\Phi\})],
$$

where $\mathscr{S}$ is defined as $\int_{0}^{\beta} d \tau \int d \mathbf{x}\left[\bar{\Phi}_{\sigma} \partial_{\tau} \Phi_{\sigma}+\mathscr{H}\right]$. A detailed description of the formulation can be found in [21]. Here we present a brief sketch for the sake of completeness. After introducing the pairing field $(\Delta(\mathbf{x}, \tau))$ and its fluctuation $\delta \Delta(\mathbf{x}, \tau)$ about the homogeneous value $(\Delta)$ in Eq. (2), the Hubbard-Stratonovich transformation is carried out. Hence one can write the effective action in terms of the Nambu propagator $\mathscr{G}^{-1}(\mathbf{x}, \tau)$. The disorder and fluctuations enter the self-energy term as $\Sigma=-\mathscr{V}_{d} \sigma_{z}+\delta \Delta \sigma_{+}+\delta \Delta \sigma_{-}$. Thus, the Nambu propagator can be presented in the following way, $\mathscr{G}^{-1}=\mathscr{G}_{0}^{-1}+\Sigma$, where $\mathscr{G}_{0}^{-1}=-\partial_{\tau} I+\left(\nabla^{2} / 2 m+\right.$ u) $\sigma_{z}+\Delta \sigma_{x}$ is the Green's function without disorder and fluctuations. Here $\sigma_{i}$ are the Pauli and ladder matrices $(i \in$ $\{x, y, z,+,-\})$. After integrating the fermionic fields, the effective action becomes

$\mathscr{S}_{\text {eff }}=\int d \mathbf{x} \int_{0}^{\beta} d \tau\left[\frac{|\Delta(\mathbf{r})|}{g}-\frac{1}{\beta} \operatorname{Tr} \ln \left\{-\beta \mathscr{G}^{-1}(\mathbf{r})\right\}\right]$,

where $\mathbf{r}=(\mathbf{x}, \tau)$. It is possible to write the effective action in Eq. (3) as a sum of bosonic action $\left(\mathscr{S}_{\mathrm{B}}\right)$ and fermionic action $\left(\mathscr{S}_{\mathrm{F}}\right)$ by expanding the inverse Nambu propagator up to the second order in self-energy (the lowest order in disorder strength). Also it contains an additional term, which emerges from the linear order of self-energy expansion $\left(\mathscr{G}_{0} \Sigma\right)$. We can set the linear order to zero if we consider $\mathscr{S}_{\mathrm{F}}$ to be an extremum of $\mathscr{S}_{\text {eff }}$ after performing all the fermionic Matsubara frequency sums. The constrained condition leads to the BCS gap equation which, after appropriate regularization through the $s$-wave scattering length, reads [22]

$-\frac{m}{4 \pi a}=\sum_{k}\left[\frac{1}{2 E_{k}}-\frac{1}{2 \varepsilon_{k}}\right]$.

This suggests that the BCS gap equation does not have any contribution from the disorder potential. The detailed calculation reveals that the disorder effect is embedded inside the density equation through the bosonic thermodynamic potential as $n_{\mathrm{B}}=-\frac{\partial \Omega_{\mathrm{B}}}{\partial \mu}$. Hence the final mean-field density equation is

$n=\sum_{k}\left(1-\frac{\xi_{k}}{E_{k}}\right)-\frac{\partial \Omega_{\mathrm{B}}}{\partial \mu}$,

where $\Omega_{\mathrm{B}}$ can be described in two parts. One contribution comes from the fluctuation of the pairing fields and the other from the disorder. The fluctuation contribution becomes significant at finite temperature. Since we specialize at zero temperature in this work, from this point on we will consider only the disorder contribution which is defined as

$\Omega_{\mathrm{B}_{d}}=\frac{\kappa}{2} \sum_{\mathbf{q}, v_{m}=0} \mathscr{N}^{\dagger} \mathscr{M}^{-1} \mathscr{N}$.

In Eq. (6), the $\mathscr{N}$ is a doublet which couples the disorder to the fluctuations. At $T=0$, after performing the fermionic Matsubara frequency summation, one finds

$\mathscr{N}_{1}=\mathscr{N}_{2}=\sum_{k} \frac{\Delta\left(\xi_{k}+\xi_{k+q}\right)}{2 E_{k} E_{k+q}\left(E_{k}+E_{k+q}\right)}$.

The inverse fluctuation propagator is a $2 \times 2$ symmetric matrix and at zero temperature it reads 


$$
\begin{aligned}
\mathscr{M}_{11}= & \frac{1}{g}+\sum_{k}\left[\frac{v_{k}^{2} v_{k+q}^{2}}{i v_{m}-E_{k}-E_{k+q}}-\frac{u_{k}^{2} u_{k+q}}{i v_{m}+E_{k}+E_{k+q}}\right], \\
\mathscr{M}_{12}= & \sum_{k} u_{k} v_{k} u_{k+q} v_{k+q} \\
& \times\left[\frac{1}{i v_{m}+E_{k}+E_{k+q}}-\frac{1}{i v_{m}-E_{k}-E_{k+q}}\right],
\end{aligned}
$$

with $\mathscr{M}_{22}(q)=\mathscr{M}_{11}(-q)$ and $\mathscr{M}_{21}(q)=\mathscr{M}_{12}(q)$. The other quantities are given in the usual BCS notation $\xi_{k}=$ $\mathbf{k}^{2} / 2 m-\mu, E_{k}=\sqrt{\xi_{k}^{2}+\Delta^{2}}, u_{k}^{2}=1 / 2\left(1+\xi_{k} / E_{k}\right)$, and $v_{k}^{2}=1 / 2\left(1-\xi_{k} / E_{k}\right)$.

\section{Results and Discussion}

Equations (4) and (5) are now ready to be solved selfconsistently, together with (7) and (8). It is clear that the disorder strength $\kappa$ is an input parameter; this initiates the question about how to estimate the disorder strength as weak. Firstly, $\kappa$ has a dimension of $k_{\mathrm{F}} / \mathrm{m}^{2}$, so in our analysis, we define the dimensionless disorder strength as $\eta=\kappa m^{2} / k_{\mathrm{F}}$. But a more physical description can be worked out if the impurity strength is normalized by Fermi density and square of Fermi energy [16]. According to the this new definition, $\kappa n_{\mathrm{F}} / \varepsilon_{\mathrm{F}}^{2}=4 /\left(3 \pi^{2}\right) \eta$. Thus, we calculate self-consistently the basic mean field quantities $\Delta$ and $\mu$ being inside this limit for different values of $\eta$.

Figure 1 demonstrates that on the BCS side the disorder does not have a significant influence. As a result the order parameter with different disorder strengths follows the mean-field approximation of $\Delta / \varepsilon_{\mathrm{F}}=8 e^{-2} \exp \left[-\pi /\left(2 k_{\mathrm{F}} a\right)\right]$ as $1 / k_{\mathrm{F}} a \rightarrow-\infty$, thus revealing the validity of Anderson theorem [4], where the weak disorder is unable to break the strongly coupled amplitude and phase coherence of the pairing field.

In the BEC limit, it is possible to make analytic extension for the bosonic thermodynamic potential by expanding the

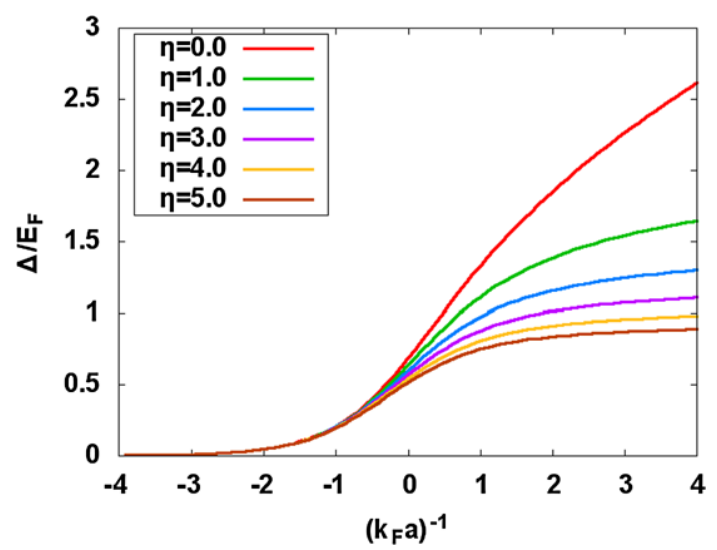

Fig. 1 The order parameter $\Delta$ scaled by the Fermi energy $E_{\mathrm{F}}$ as a function of $\left.1 / k_{\mathrm{F}} a\right)$ and for various values of the disorder strength inverse fluctuation propagator matrix elements in powers of $q$ [23]. A systematic and careful calculation leads to an analytic description of the order parameter in this limit, where one can observe a progressive depletion. Here the impurity potential actually starts to destroy the superfluidity and, in effect, the order parameter is depleted and the fraction of depletion remains of the order of $\eta /\left(k_{\mathrm{F}} a\right)$ which is in agreement with [15]. Hence Anderson theorem breaks down in this limit. The other mean-field quantity, the chemical potential, remains pinned to the Fermi energy in the BCS limit and follows the ideal path (without disorder) towards the BEC limit, as seen Fig. 2. We understand that it might be an attribute of the fluctuation theory [24], where the correction in $\mu$ at the BCS side is usually in $\mathscr{O}\left(\Delta^{2}\right)$ which is a very small quantity as $\Delta \rightarrow 0$ for $1 /\left(k_{\mathrm{F}} a\right) \rightarrow 0$. On the BEC side, the correction comes through the effective chemical potential of the composite bosons. However, the BEC chemical potential is dominated by the binding energy, and it turns out to be quite large compared to the effective chemical potential (therefore, a more relevant normalization in BEC limit can be binding energy which leads to saturation of the chemical potential at -1 . However, to remain consistent, we presented both $\Delta$ and $\mu$ in the units of Fermi energy). Hence the chemical potential remain almost unchanged.

In the crossover window (which is usually considered as $\left.1 /\left(k_{\mathrm{F}} a\right) \simeq[-1,1]\right)$, one can now find the distinguishing features of the order parameter (see Fig. 1) as a function of disorder strength. Thus, from this figure one can clearly say that the disorder starts to play its role in the crossover. At this point, it is preferable to comment on the role of order parameter and disorder strength. As mentioned above, we work in the weak disorder limit, therefore it can be considered as homogeneous or quasi-homogeneous and the formalism of pairing fluctuation suits well. In this limit, the local pairing amplitude $\left(\left\langle c_{\downarrow} c_{\uparrow}\right\rangle\right)$ is same as the off-diagonal long-range order (ODLRO) $\left(\sqrt{\left\langle c_{\uparrow}^{\dagger} c_{\downarrow}^{\dagger} c_{\downarrow} c_{\uparrow}\right\rangle}\right)$ and the spectral gap. Thus, from Fig. 1 it can be concluded that the random impurity

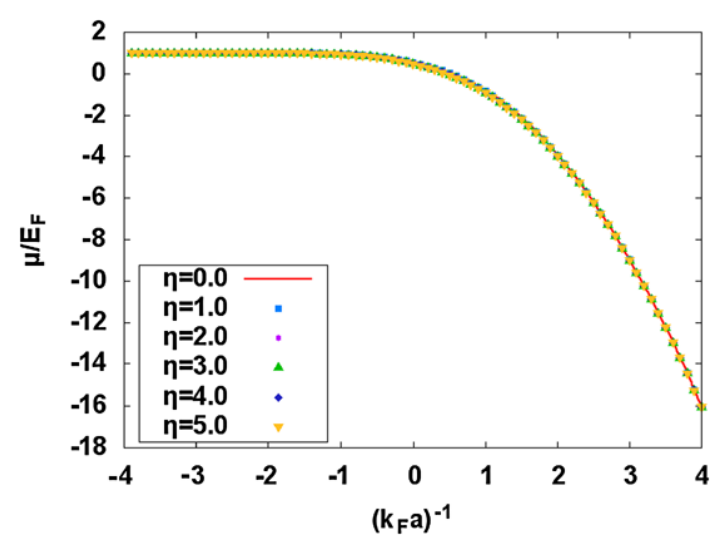

Fig. 2 The chemical potential $\mu$ scaled by the Fermi energy $E_{\mathrm{F}}$ as a function of $1 /\left(k_{\mathrm{F}} a\right)$ and for various values of the disorder strength 


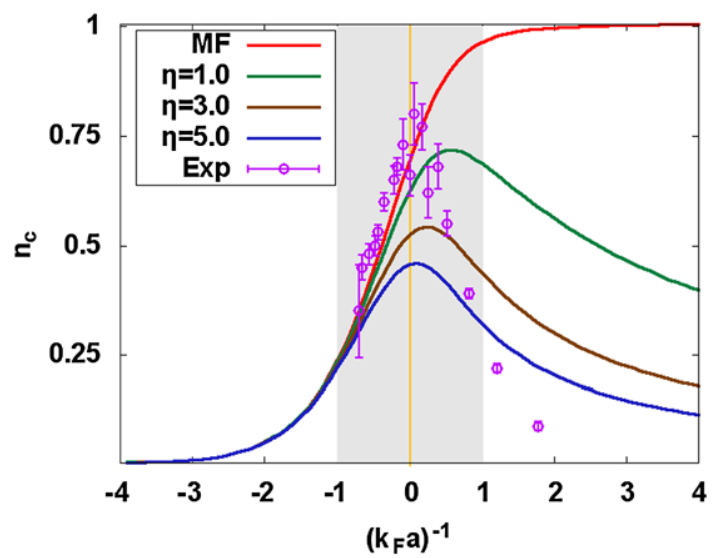

Fig. 3 The condensate fraction shows a non-monotonic behavior in the presence of disorder. The red line represents the mean field result in clean Fermi gas and the open circles represents the condensate fraction data from $[20,25]$

starts to destroy the ODLRO even in the strongly correlated crossover region. The story becomes completely different when the disorder is strong as the condition of homogeneity will break. In a practical scenario, for $s$-wave superconductors, the distribution of the local pairing amplitude looses spatial uniformity, the ODLRO goes to zero and the spectral gap increases non-monotonically [11]. In this work, the weak disorder consideration simplifies the picture, but we can still get a glimpse of the impurity driven situation where the destruction of ODLRO can be viewed clearly.

The order parameter and the chemical potential are not directly measurable, thus we switch our attention to a more experimentally viable quantity, the condensate fraction $\left(n_{c}\right)$. In a clean Fermi gas, it is possible to work out the condensate fraction through mean-field theory [25] which shows good agreement with the experiment [20]. Here we follow a similar mean-field description

$n_{c}=\sum_{k}\left[\frac{\Delta(\eta)}{2 E_{k}(\eta)}\right]^{2}$.

The only difference with the clean system calculation is that in Eq. (9) we used the disorder induced values for $\Delta$ and $\mu$. In Fig. 3, we present the mean-field result for the clean Fermi gas, the disorder induced condensate fraction, and the experimental data for the clean Fermi gas $\left({ }^{6} \mathrm{Li}\right)$ [20]. As one expects, the condensate fraction decays similar to that of the clean limit when $1 /\left(k_{\mathrm{F}} a\right) \rightarrow-\infty$, as $n_{c} \propto \Delta$ and $\Delta$ does not change with the variation of $\eta$ in the BCS regime. However, if one extends the self-energy up to the second order in the condensate fraction calculation, one can observe the effect of disorder in the BCS limit [15]. In this limit, the experimental data, theoretical clean Fermi gas calculation, and the disorder modified results show good agreement with each other.
On the BEC side, the disorder destroys part of the condensate and turns it into a normal fluid. The condensate fraction approaches roughly $\eta / \sqrt{k_{\mathrm{F}} a}$ as obtained from the study of hard-sphere Bose gas in random disorder [13]. Interestingly, we also observe a decay of the condensate fraction in the experimental data. It is explained as the result of inelastic losses for the more tightly bound molecules [20]. In our model, we have considered an elastic scattering process through a static impurity, but we guess this model itself renders some information about the loss in condensate fraction in the BEC side.

The non-monotonic behavior of the condensate fraction in the crossover region (grey area in Fig. 3) is the most intriguing point. One can see a significant amount of condensate fraction in this region in comparison to the two extremes of BCS and BEC sides. The peak of the condensate fraction for the experimental data and disordered Fermi gas lies in the region $1 /\left(k_{\mathrm{F}} a\right) \rightarrow 0^{+}$. Thus, the experimental results and a simple model with quenched disorder qualitatively show similar features. In both cases, the loss of the condensate fraction is due to additional scattering process. That the most stable region is around unitarity may be regarded as a signal towards a relatively robust paradigm of superfluidity [26].

\section{Conclusion}

In conclusion, we have included an impurity like weak disorder via the Gaussian fluctuation route and then solved the coupled BCS mean-field equations self-consistently as a function of the inter-particle interaction (controlled by $k_{\mathrm{F}} a$ ) to address the BCS to BEC crossover ultracold Fermi gases. This enabled us to obtain the two basic mean-field parameters $\Delta$ and $\mu$ where we show that the order parameter is depleted but the chemical potential is unchanged. The gradual decrease of $\Delta$ can be connected to the destruction of superfluidity. It is already known [13] that the random potential destroys the superfluid nature in Bose gas, hence the superfluid order parameter gets depleted. Also it shows that the destructive process begins around the resonance position. It will be very interesting to study the crossover region under the influence of a large impurity as it is still an unexplored domain. Further, we qualitatively compared the disorder induced condensate fraction with the experimental result for a clean Fermi gas. In both cases, the condensate fraction follows the mean-field prediction in the BCS side whereas in the BEC side it exhibits a drop. At unitarity, both show a maximum of condensate fraction which might be connected to the more stable region of superfluidity of the strongly correlated fermionic pairs. Our calculations thus are in good qualitative agreement with the experimental results. Therefore, we believe that a comprehensive weak disorder model can shed some light to the Fermi gases in the BCS-BEC crossover. 
Acknowledgements This work is supported by TUBITAK (Grants No. 109T267, 210T050, 209T050) and TUBA. AK acknowledges the visiting program in IISER-Kolkata and P.K. Panigrahi. SB acknowledges DST grant number SR/S2/CMP/0023/2009.

\section{References}

1. Chin, C., Grimm, R., Julienne, P., Tiesinga, E.: Rev. Mod. Phys. 82, 1225 (2010)

2. Giorgini, S., Pitaevskii, L.P., Stringari, S.: Rev. Mod. Phys. 80, 1215 (2008)

3. Bloch, I., Dalibard, J., Zwerger, W.: Rev. Mod. Phys. 80, 885 (2008)

4. Anderson, P.W.: J. Phys. Chem. Solids 11, 26 (1959)

5. Billy, J., et al.: Nature 453, 891 (2008)

6. Roati, G., et al.: Nature 453, 895 (2008)

7. Kondov, S.S., McGhee, W.R., Zirbel, J.J., DeMarco, B.: Science 334, 66 (2011)

8. Jendrzejewski, F., et al.: Nat. Phys. 8, 398 (2012)

9. Palencia, L.S., Lewenstein, M.: Nat. Phys. 6, 87 (2010)

10. Belitz, D., Kirkpatrick, T.R.: Rev. Mod. Phys. 66, 261 (1994)

11. Ghoshal, A., Randeria, M., Trivedi, N.: Phys. Rev. B 65, 014501 (2001)
12. Bouadim, K., Loh, Y.L., Randeria, M., Trivedi, N.: Nat. Phys. 7, $884(2011)$

13. Huang, K., Meng, H.F.: Phys. Rev. Lett. 69, 644 (1992)

14. Giorgini, S., Pitaevskii, L., Stringari, S.: Phys. Rev. B 49, 12938 (1994)

15. Orso, G.: Phys. Rev. Lett. 99, 250402 (2007)

16. Han, L., Sá de Melo, C.A.R.: New J. Phys. 13, 055012 (2011)

17. Dey, P., Basu, S.: J. Phys. Condens. Matter 20, 485205 (2008)

18. Khan, A., Basu, S., Kim, S.W.: J. Phys. B, At. Mol. Opt. Phys. 45, 135302 (2012)

19. Nozières, P., Schmitt-Rink, S.: J. Low Temp. Phys. 59, 195 (1985)

20. Zwierlein, M.W., et al.: Phys. Rev. Lett. 92, 120403 (2004)

21. Khan, A.: Int. J. Mod. Phys., Conf. Ser. 11, 120 (2012)

22. Griffin, A., Snoke, D.W., Stringari, S. (eds.): Bose-Einstein Condensation. Cambridge Univ. Press, Cambridge (1995)

23. Taylor, E., Griffin, A., Fukushima, N., Ohashi, Y.: Phys. Rev. A 74, 063626 (2006)

24. Diener, R.B., Sensharma, R., Randeria, M.: Phys. Rev. A 77, 023626 (2008)

25. Salasnich, L., Manini, N., Parola, A.: Phys. Rev. A 72, 023621 (2005)

26. Zwierlein, M.W. et al., Nature 435, 1047 (2005) 